\title{
CRTAP mutations in lethal and severe osteogenesis imperfecta: the importance of combining biochemical and molecular genetic analysis
}

Fleur S Van Dijk ${ }^{\star}, 1$, Isabel M Nesbitt ${ }^{2}$, Peter GJ Nikkels ${ }^{3}$, Ann Dalton ${ }^{2}$, Ernie MHF Bongers ${ }^{4}$, Jiddeke M van de Kamp ${ }^{1}$, Yvonne Hilhorst-Hofstee ${ }^{5}$, Nicolette S Den Hollander ${ }^{5}$, Augusta MA Lachmeijer ${ }^{1}$, Carlo L Marcelis ${ }^{4}$, Gita MB Tan-Sindhunata ${ }^{1}$, Rick R van Rijn ${ }^{6}$, Hanne Meijers-Heijboer ${ }^{1}$, Jan M Cobben ${ }^{7}$ and Gerard Pals ${ }^{1}$

\footnotetext{
${ }^{1}$ Department of Clinical Genetics, VU University Medical Cen, Amsterdam, The Netherlands; ${ }^{2}$ Sheffield Molecular Genetics Service, Sheffield Children's Hospital NHS Foundation Trust, Sheffield, United Kingdom; ${ }^{3}$ Department of Pathology, University Medical Centre, Utrecht, The Netherlands; ${ }^{4}$ Department of Human Genetics, Radboud University Nijmegen Medical Centre, Nijmegen, The Netherlands; ${ }^{5}$ Department of Clinical Genetics, Leiden University Medical Centre, Leiden, The Netherlands; ${ }^{6}$ Department of Pediatric Radiology, Academic Medical Centre, Amsterdam, the Netherlands; ${ }^{7}$ Department of Pediatrics, Academic Medical Centre, Amsterdam, The Netherlands
}

Autosomal recessive lethal and severe osteogenesis imperfecta (OI) is caused by the deficiency of cartilageassociated protein (CRTAP) and prolyl-3-hydroxylase 1 (P3H1) because of CRTAP and LEPRE1 mutations. We analyzed five families in which 10 individuals had a clinical diagnosis of lethal and severe OI with an overmodification of collagen type I on biochemical testing and without a mutation in the collagen type I genes. CRTAP mutations not described earlier were identified in the affected individuals. Although it seems that one important feature of autosomal recessive OI due to CRTAP mutations is the higher consistency of radiological features with OI type II-B/III, differentiation between autosomal dominant and autosomal recessive OI on the basis of clinical, radiological and biochemical investigations proves difficult in the affected individuals reported here. These observations confirm that once a clinical diagnosis of OI has been made in an affected individual, biochemical testing for overmodification of collagen type I should always be combined with molecular genetic analysis of the collagen type I genes. If no mutations in the collagen type I genes are found, additional molecular genetic analysis of the CRTAP and LEPRE 1 genes should follow. This approach will allow proper identification of the genetic cause of lethal or severe OI, which is important in providing prenatal diagnosis, preimplantation genetic diagnosis and estimating recurrence risk.

European Journal of Human Genetics (2009) 17, 1560-1569; doi:10.1038/ejhg.2009.75; published online 24 June 2009

Keywords: osteogenesis imperfecta; recessive; CRTAP; collagen type 1

Introduction

Osteogenesis imperfecta (OI) was first described by the Swedish surgeon, Olaus Jakob Ekman, in a family with

${ }^{*}$ Correspondence: Dr FS van Dijk, Department of Clinical Genetics, De Boelelaan 1117, $1081 \mathrm{HV}$, Amsterdam, The Netherlands.

Tel: + 3120 4440150; Fax: + 3120 4440769;

E-mail: fs.vandijk2@vumc.nl

Received 21 October 2008; revised 29 January 2009; accepted 29 March 2009; published online 24 June 2009 hereditary bone fragility (1788). ${ }^{1}$ On account of the extreme variability in the presentation of OI, a classification into four types (OI type I (mild), II (lethal), III (severe) and IV (moderate) was introduced by Sillence et al (1979) (the 'Sillence classification')). Type II OI was further subdivided into type $\mathrm{A}, \mathrm{B}$ and $\mathrm{C}$ on the basis of radiological features. The earlier assumption based on pedigree findings that OI type II was inherited in an autosomal recessive way shifted toward the view that OI type II was primarily 
inherited as an autosomal dominant disease $\mathrm{e}^{2-5}$ with the discovery of heterozygous mutations in the collagen type I genes, COL1A1 and COL1A2, in all types of OI. In addition, it was recorded that most recurrences in OI type II and III were caused by gonadal mosaicism in one of the parents. The empirical recurrence rate of lethal OI was estimated at $6 \% .^{6-10}$ However, it seemed that not all patients with lethal or severe OI had a collagen type I mutation. ${ }^{11,12} \mathrm{~A}$ hypomorphic loss-of-function of CRTAP-encoding cartilage-associated protein (CRTAP) was shown to cause the rhizomelic form of moderate-severe recessive OI, originally described as OI type VII. ${ }^{13,14}$ Furthermore, complete loss-of-function mutations of CRTAP and of LEPRE1, which encode CRTAP and prolyl-1-hydroxylase (P3H1), seemed to cause autosomal recessive lethal and severe OI. ${ }^{14,15,16}$ CRTAP, P3H1 and cyclophilin B (CyPB) encoded by PPIB form a protein complex in the rough endoplasmic reticulum (rER), which is responsible for 3-hydroxylation of proline at position 986 in the $\alpha 1$ chain of collagen type I. In individuals affected with lethal or severe OI due to mutations in COL1A1, COL1A2, CRTAP and LEPRE1, comparable delayed migration of collagen type I is evident, which is visible as backstreaking, increased band width or baseline shift on electrophoresis (see Figure 1). This phenomenon is due to posttranslational overmodification and can be explained by the structure of collagen type I, which is first synthesized as procollagen type I consisting of two pro $\alpha 1$ (I) chains and one pro $\alpha 2(\mathrm{I})$ chain, containing
$\mathrm{N}$ - and C-terminal propeptides. Posttranslational modification necessary to reach intertwining of the chains resulting in triple-helical conformation takes place in the rER. Currently, two posttranslational modification systems of collagen type I are known, namely, (i) hydroxylation of multiple lysine and proline residues by lysyl hydroxylase and prolyl-4-hydroxylase and (ii) 3-hydroxylation of a single residue in the $\alpha 1$ chain, proline at position 986 by the protein complex in the rER, consisting of P3H1, CRTAP and CyPB. Triple-helical folding occurs in the C-to-N direction, making lysines and prolines inaccessible to posttranslational modification as soon as the chain in which they are located is folded. Posttranslational overmodification takes place when a delay in triple-helical folding results in overprocessing of the constituent collagen type I chains by the enzymes involved in posttranslational modification. This delay can be caused by collagen type 1 mutations disturbing the primary structure of collagen type I or by deficiency in two of the components of the prolyl-3-hydroxylation complex, CRTAP and P3H1 LEPRE1. ${ }^{17-20}$ Here, we describe 10 individuals affected with lethal or severe OI due to novel CRTAP mutations.

\section{Materials and methods \\ Patients}

We selected 13 individuals from seven families for screening, on the basis of clinical diagnosis of OI type II/
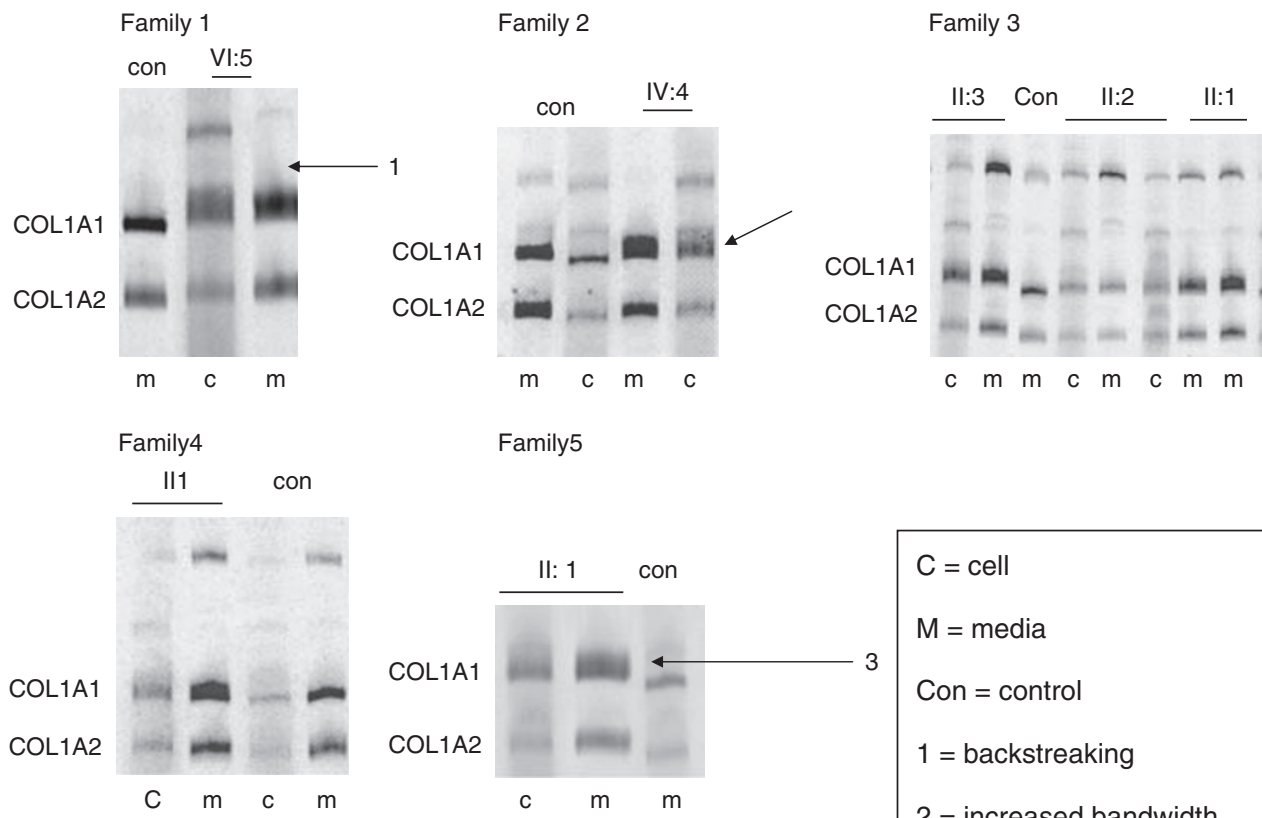

Family5

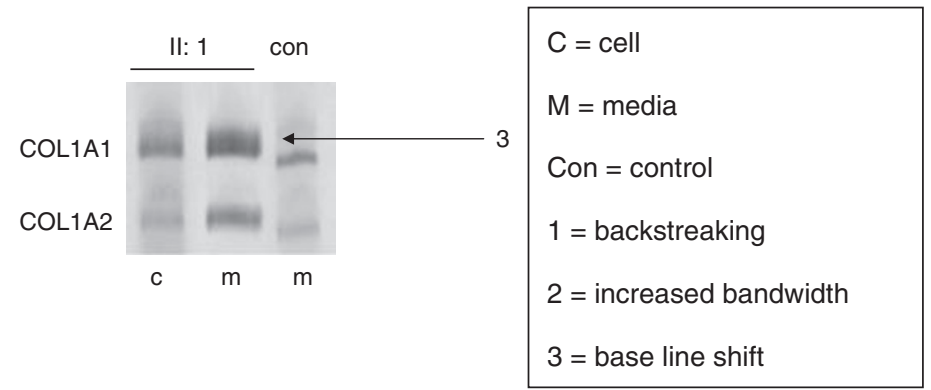

Figure 1 Biochemical testing in families 1-5. Overmodification on electrophoresis is evident by either backstreaking and/or baseline shift and/or increased band width. Three examples of these biochemical features are numbered in the electrophoresis images of the families. 
III, collagen type I overmodification on electrophoresis and absence of collagen type I mutations.

\section{Protein analysis}

Skin biopsies were obtained from the affected individuals, and fibroblast cultures were established under standard conditions. Cells were seeded at 35000 cells $/ \mathrm{cm}^{2}$. Labeling of the fibroblasts and purification of the collagen molecules were carried out as reported elsewhere, excluding the carrying out of SDS electrophoresis without 3\% stacking gel and exposing the dried gels to a phosphor imager screen (Molecular Dynamics, Sunnyvale, CA, USA). ${ }^{21}$

\section{DNA analysis of the collagen type 1 genes}

cDNA of the COL1A1 and COL1A2 genes was prepared from total RNA from cultured fibroblasts. RNA was isolated using the RNA Isolation Minikit (Qiagen, Hilden, Germany), according to the manufacturer's instructions. Full-length single-stranded cDNA was prepared with oligodT-primer and Superscript II RT Reverse Transcriptase (Invitrogen, San Diego, CA, USA). The complete coding sequences of the COL1A1 and COL1A2 genes (reference sequence NM_000088 and NM_000089) were both analyzed by direct sequencing in 11 overlapping PCR fragments (primer sequences available on request).

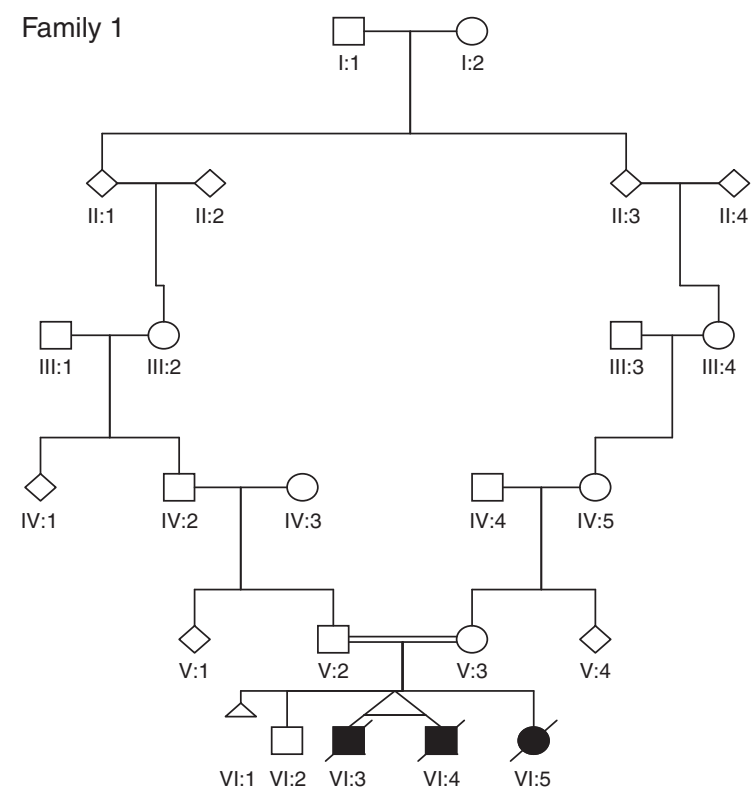

Family 3

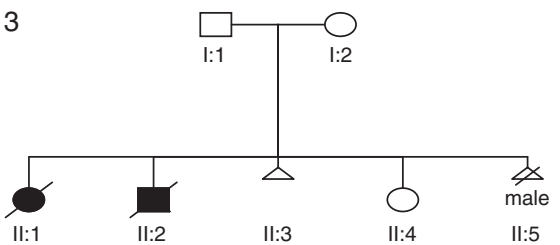

Molecular analysis of the CRTAP and LEPRE1 genes Molecular analysis of CRTAP (reference sequence NM_006371), LEPRE1 (reference sequence NM_022356) and PPIB was carried out as reported elsewhere. ${ }^{14,15,21}$

\section{Results}

In all 13 individuals from seven families, posttranslational overmodification on electrophoresis was evident. In 2 individuals from one family, LEPRE1 mutations were detected, in 1 individual no mutations in CRTAP, LEPRE1 and $P P I B$ were detected and 10 individuals from five families appeared to have novel CRTAP mutations. Here, we describe the clinical features and mutation analysis of patients with OI due to CRTAP mutations only.

\section{Family 1}

Clinical findings The parents were consanguineous (Caucasian) and the mother was pregnant with male monozygotic twins (Figure 2). An advanced ultrasound screening at 21 weeks of gestation showed bowed and short femura $<$ p5 in both fetuses (VI:3 and VI:4). In one fetus, a possible fracture of the femur was seen. Owing to the strong suspicion of OI type II/III, the pregnancy was terminated at $23+2$ weeks of gestation. No permission was given for autopsy. On the babygram, fractures of the
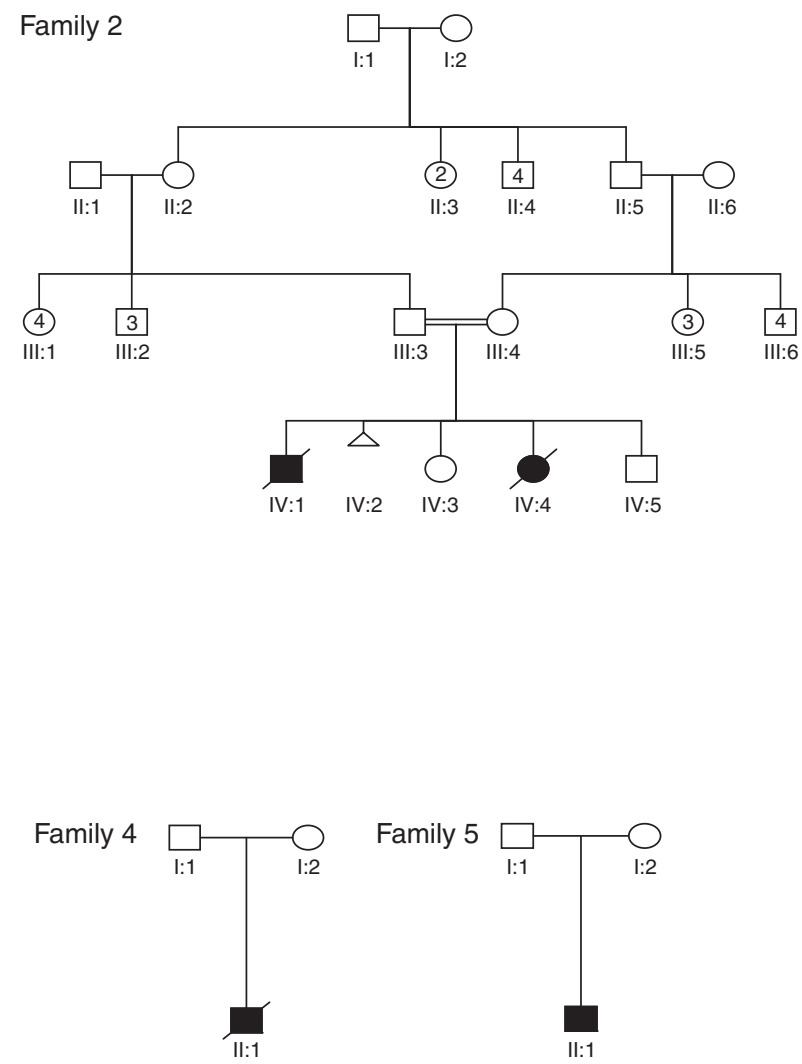

Figure 2 Pedigree families 1-5. 
femora were detected in both fetuses, with no apparent rib fractures (Supplementary Appendix Figures 3 and 4). The fetuses were diagnosed with OI type II-B.

In the following pregnancy, an advanced ultrasound in the 19th week of gestation again showed skeletal abnormalities consisting of a fracture of the femur, a bowed tibia and short osseous elements. The parents decided to proceed with the pregnancy, and a daughter (VI:5) was born by cesarean section at 39 weeks of gestation, with an Apgar score of 9, 9 and 10 after 1, 5 and $10 \mathrm{~min}$, respectively. Birth weight was $3245 \mathrm{~g}$. The neonate had normocephaly, a round face with shallow orbits, white sclerae, long philtrum, small thorax, rhizomelic shortening of the upper and lower extremities and abducted position of the legs (Figure 3). A skeletal overview showed evidence of multiple fractures (Figure 4). The girl was diagnosed with OI type III and is alive at the age of 2 years.

Mutation analysis In the twin, monozygosity was confirmed by marker studies. Mutation screening of the COL1A1 and COL1A2 genes in the fetuses, VI:3 and VI:4, identified a variant, c.613 C>G; p.pro205Ala, in the COL1A1 gene. This variant has been reported in literature before and is probably associated with osteopenia. ${ }^{23}$ Mutation analysis of the ALPL gene responsible for hypophosphatasia was negative. In the third affected child, no mutations in the COL1A1,COL1A2 and LEPRE1 genes were found.

Sequencing of the CRTAP gene in the three affected children revealed homozygosity for the mutation, c.21_22dupGG, introducing a premature termination codon at p.Ala8fs in exon 1 . The parents were heterozygous for this mutation.

\section{Family 2}

Clinical findings The parents were consanguineous (Moroccan). In the third trimester of pregnancy, extensive skeletal abnormalities were observed in the fetus. A boy (IV:1) was born with multiple fractures, consistent with the diagnosis of OI type II/III. He died 1 month after birth (Figure 2).

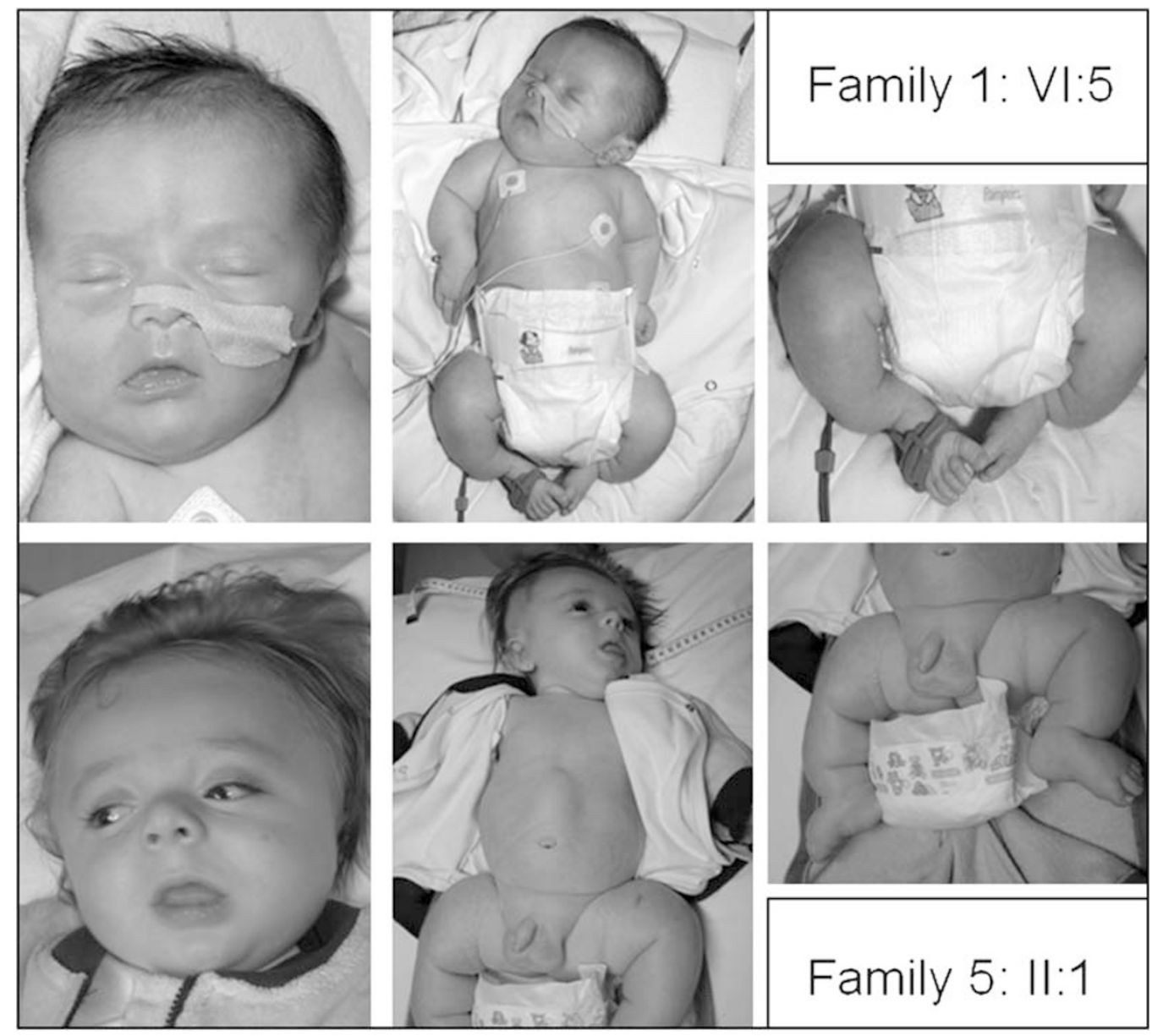

Figure 3 Clinical assessments of family 1 VI: 5 and family 5 II:1. Note round face with shallow orbits, white or grayish sclerae, long philtrum, small thorax, rhizomelic shortening of the upper and lower extremities and abducted position of the legs. 

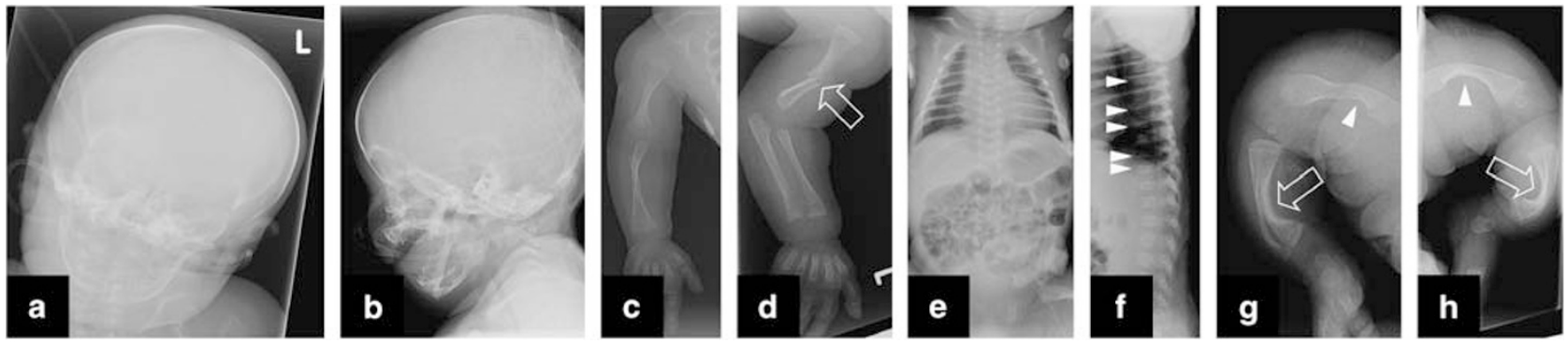

Figure 4 Radiographs of individual VI:5 from family 1. (a) Apparent normal mineralization of the skull, no Wormian bones. (b) Mild brachycephaly. (c) Normal aspect of the right arm. A rhizomelic shortening may exist that may also be because of projection, given the normal length of the left humerus. (d) Oblique mid diafyseal fracture of left humerus (arrow). (e) Normal chest radiograph without rib fractures. (f) Platyspondyly of thoracic vertebrae, 5, 7, 8, 10 and 11 (arrowheads). (g) Bowing of femora (arrowhead), tibiae and fibulae (arrow). (h) Bowing of femora (arrowhead), tibiae and fibulae (arrow).
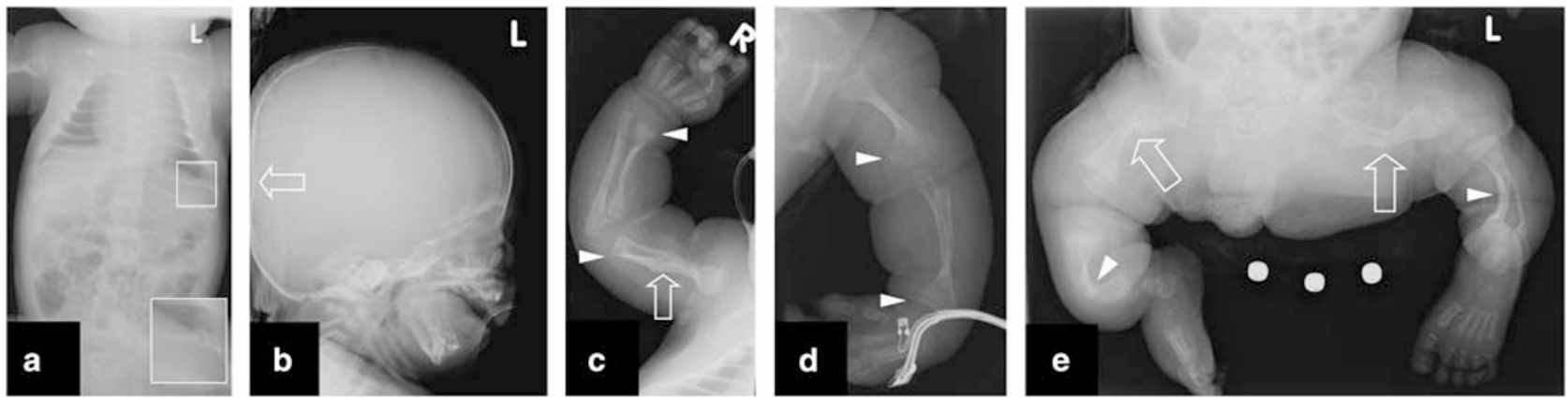

Figure 5 Radiographs of individual IV:4 from family 2. (a) Small thorax. Slender ribs. Lateral fracture of the eighth left rib (see insert). Asymmetric platyspondyly, Th5 and Th7 (visible on original radiograph). (b) Slight flattening of the skull (arrow). (c) Consolidation of the right humerus fracture (arrow). Humerus measures $4.38 \mathrm{~cm}$ (normal range $=6.0-7.7 \mathrm{~cm}$.) and the ulna $4.9 \mathrm{~cm}$ (normal range $=5.8-7.2 \mathrm{~cm}$.). Broad distal metaphyses of the left humerus and radius (arrowhead). Possible fracture of the left radius. (d) Broad distal metaphyses of the left humerus and ulna (arrowhead). (e) Bilateral proximal femur fracture, with consolidation and angulation (arrows). Bilateral bowing of tibia and fibula (arrowheads).

In the fourth pregnancy, ultrasound images around the 20th week of gestation displayed skeletal abnormalities suggestive of OI. At $23+2$ weeks of gestation, shortened and deformed extremities, a fracture of the left femur and a bell-shaped thorax were observed on ultrasound screening. A girl (IV:4) was born at $38+3$ weeks. A skeletal overview showed gracile bones and multiple fractures with callus formation (Figure 5). The girl died 1 week later because of respiratory insufficiency. A femur was taken for histological examination (Figure 6).

Mutation analysis Fibroblast cultures of the first child became infected, and no mutations in the COL1A1, COL1A2 and LEPRE1 genes were identified in the second affected child. Sequencing of the CRTAP gene showed homozygosity for the mutation, c.404delG, introducing a premature termination codon at p.Ser135fs in exon 1 of the CRTAP gene. The parents were both heterozygous for this mutation.

\section{Family 3}

Clinical findings A caucasian girl (II:1) was born at $40+6$ weeks of gestation with Apgar scores of 7 and 9 at 1 and $5 \mathrm{~min}$, respectively. On physical examination, fragmentation of the skull bones was observed and a short thorax with short and bowed extremities was noticed, suggesting OI. The child had no overt blue sclerae. A skeletal overview showed multiple rib fractures and fractures of both the upper and lower extremities. The neonate was diagnosed with OI type II-C/III. At the age of 2.5 months, she died due to respiratory failure.

In the following pregnancy, an ultrasound in the 17 th week showed no evidence of fractured or bowed extremities. However, at $18+4$ weeks of gestation, fractures of both femora were observed. A recurrence of OI was suspected and the pregnancy was terminated at $20+2$ weeks. Multiple fractures were evident (Figure 7) and the fetus (II:2) was diagnosed with OI type II (Figure 2). 

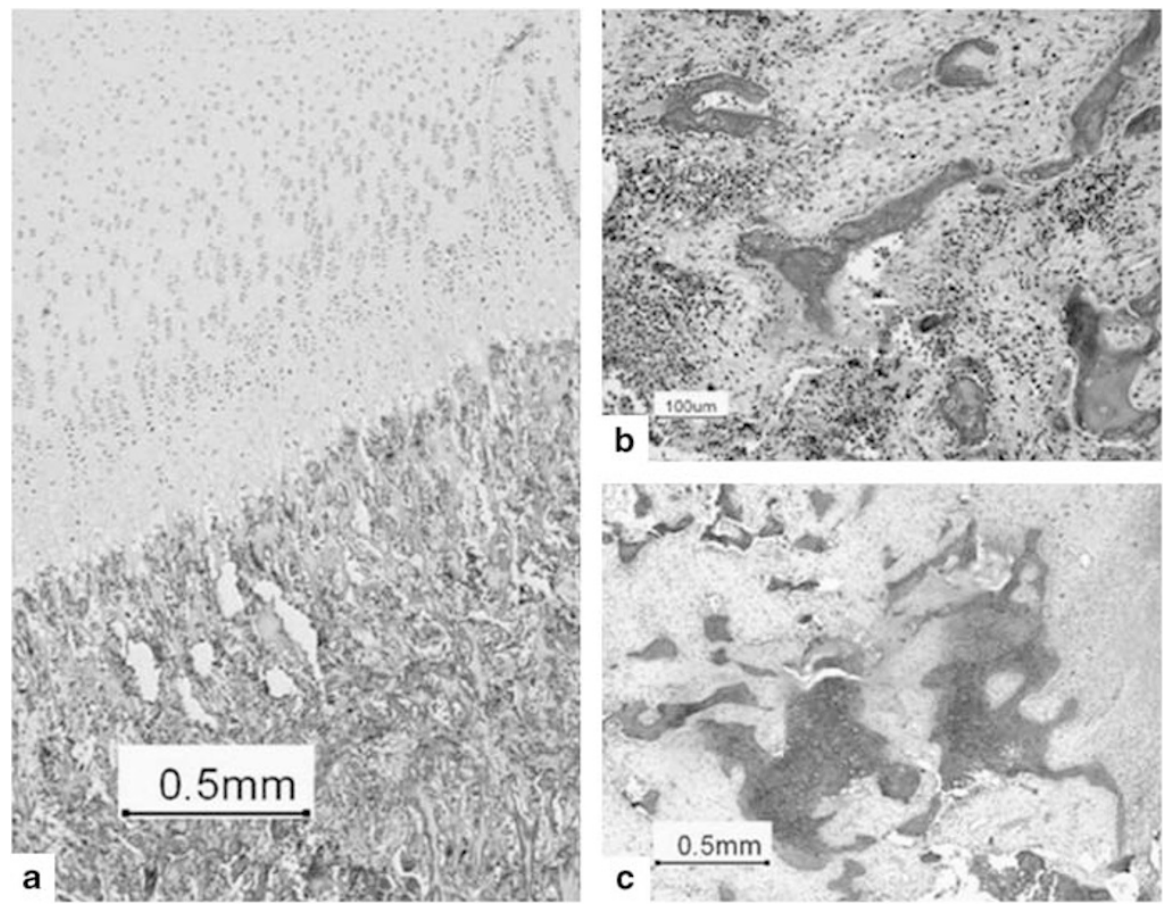

Figure 6 Bone histology of family $2 \mathrm{IV}: 4$. (a) $\mathrm{H}$ and E stain of the epiphysial growth zone of femur. Note the sharp demarcation between cartilage and primary spongiosa. The epiphysial growth zone and primary spongiosa are normal. The resting cartilage shows no abnormalities. (b) $\mathrm{H}$ and $\mathrm{E}$ stain of the metaphysis for $\mathrm{OI}$, typical small, slender and hypercellular bony trabeculae and fibrotic medulla. The bony trabeculae consist of woven bone. (c) Low-power PAS acian blue stain of the midshaft of the femur, with metaplastic cartilage formation (blue) in the area of a fracture with fibrotic marrow. This formation of cartilage is quite typical for the severe forms of Ol. ${ }^{26}$

The fifth pregnancy (II:5) was terminated because of an evident posttranslational overmodification of collagen type 1 in cultured chorion villi.

Mutation analysis Mutations in the COL1A1, COL1A2 and LEPRE1 genes were not detected in the three affected children. Sequencing of the CRTAP gene showed that they were all compound heterozygous for a c.198C $>$ A mutation predicted to result in a p.Tyr66X amino acid change in exon 1 and a c. $471+2 \mathrm{C}>\mathrm{A}$ splice-site change in intron 1 of the CRTAP gene. The mother was heterozygous for the c. $471+2 \mathrm{C}>\mathrm{A}$ mutation and the father was heterozygous for the c.198C $>$ A mutation.

\section{Family 4}

Clinical findings At a pregnancy duration of 20 weeks, ultrasound abnormalities were observed consisting of short and bowed extremities and a short thorax. Suspecting OI, a cesarean section was carried out at $39+4$ weeks of gestation. A caucasian boy (II:1) was born with Apgar scores of 2-3 and 8-9 after 1 and 5 min, respectively. He had a large anterior fontanel, short thorax, short and bowed extremities and glandular hypospadia. A skeletal overview showed wormian bones, as well as multiple fractures of ribs and of both the upper and lower extremities (Figure 8). He was diagnosed with OI type III and died 5 days after birth (Figure 2).

Mutation analysis Mutations in the COL1A1, COL1A2 and LEPRE1 genes were not detected in the affected child. Sequencing of the CRTAP gene showed that the affected child was compound heterozygous for a splice-site mutation, c.923-2A $>$ G, in intron 4 and for two mutations on the other allele in exon 1, namely, c.38C $>\mathrm{A}$ and c. $469 \mathrm{~A}>\mathrm{G}$. The mother was heterozygous for the c. $38 \mathrm{C}>\mathrm{A}$ and c. $469 \mathrm{~A}>\mathrm{G}$ mutations in exon 1 . The father was not available.

\section{Family 5}

Clinical findings In the first pregnancy, abnormalities of the extremities were observed in the fetus at 23 weeks of gestation. At the 24th week of gestation, short upper and lower extremities were observed, indicating severe skeletal dysplasia. At a gestation period of $40+4$ weeks, a caucasian boy (II:1) was born. He had a round face with shallow orbits, grayish sclerae, small thorax, rhizomelic shortening of the upper and lower extremities, abducted position of the legs and normocephaly (Figure 9). A skeletal overview showed multiple fractures, suggesting OI type II/III. The child is alive at the age of 4 years (Figure 2). 

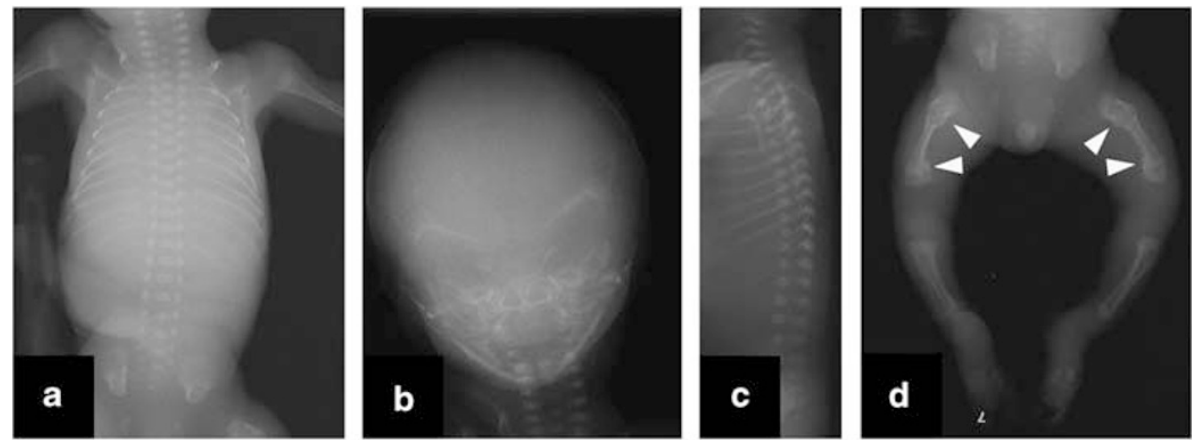

Figure 7 Radiographs of II:2 from family 3. (a) Slender ribs. No rib fractures, two fractures of right femur with callus formation. (b) Underexposed radiograph, which makes evaluation for mineralization difficult. The calvaria seem to be thin for gestational age. (c) No vertebral abnormalities. (d) Two fractures of both femora with callus formation (arrowheads).
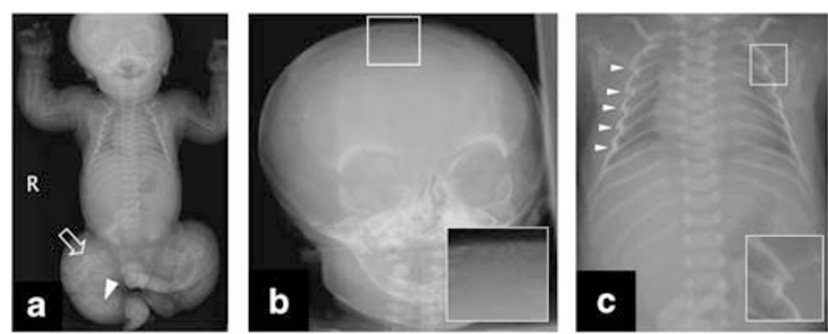

Figure 8 Radiological images of II:2 from family 4. (a) No fractures of humeri, radii and ulnae. Multiple fractures of the femora, with loss of modeling (arrow). Fracture and bowing of right tibia (arrowhead). (b) Wormian bones (see insert), broad skull. (c) Slender ribs, lateral rib fractures of the left rib 2 (see insert) and right ribs 2-6 (arrowheads).
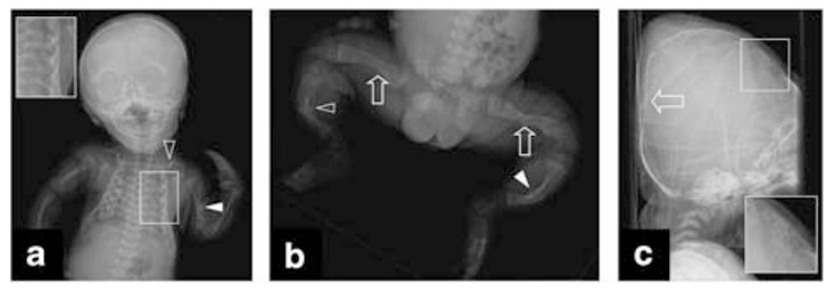

Figure 9 Radiological images of II:1 from family 5. (a) Fracture of the left clavicula (arrowhead), small thorax, normal vertebral column, fractures of left ribs 4-7 (see insert). Fractures of the left humerus (solid arrowhead) and radius. Consolidated fracture of right humerus. (b) Fracture of the right femur (arrow) and tibia (arrowhead), severe bowing of right fibula and tibia. Multiple fractures of the left femur (arrow), bowing and fracture of left tibia (solid arrowhead). (c) Wormian bones (see insert) and flattening of the occiput (arrow).

Mutation analysis Mutations in the COL1A1, COL1A2 and LEPRE1 genes were not detected in the affected child. Sequencing of the CRTAP gene showed homozygosity for the c. $471+2 \mathrm{C}>\mathrm{A}$ mutation in intron 1 of CRTAP.

\section{Discussion}

We describe 10 individuals with lethal or severe OI on the basis of clinical (Table 1 Supplementary Appendix), radiological (Table 1) and biochemical (Figure 1) findings, in whom novel CRTAP mutations (Table 2) were found. So far, six probands with lethal or severe OI due to CRTAP mutations have been reported in literature. An important question is whether the phenotype of patients with lethal OI due to CRTAP mutations differs from that of patients with OI due to mutations in the collagen type I genes.

Barnes et al, ${ }^{15}$ described in three individuals with OI due to CRTAP mutations, some clinical features in CRTAPrelated OI, which differed from the clinical features in individuals with OI due to collagen type I mutations, namely, (i) no relative macrocephaly, (ii) a round face with shallow orbits, (iii) white or grayish sclerae and (iv) rhizomelic shortening of the upper and lower extremities. Indeed, our patients, VI:5 and II: 1 from family 1 and 5 depicted in Figure 3, show no relative macrocephaly, a round face and no blue sclerae. The clinical assessments of VI:5 suggest rhizomelic shortening of the arms. However, because of the absence of sufficient clinical details of the other liveborn children with CRTAP mutations, the specificity of these clinical differences is uncertain. Therefore, a distinction between autosomal dominant OI due to collagen type I mutations and autosomal recessive OI due to CRTAP mutations on clinical features proves difficult.

Radiologically, bowing of extremities and/or fractures could be observed in the prenatal and neonatal period in all our patients with CRTAP mutations. The earliest appearance of features of OI was noted in the 18th week of pregnancy (Table 1 Supplementary Appendix). However, this pregnancy was monitored extensively because of an affected sib. Recently, 'popcorn' epiphyses have been suggested to be a distinguishing radiological feature of autosomal recessive OI, reflecting a cartilaginous dysplasia at the developing growth plate. ${ }^{22}$ However, histology of 


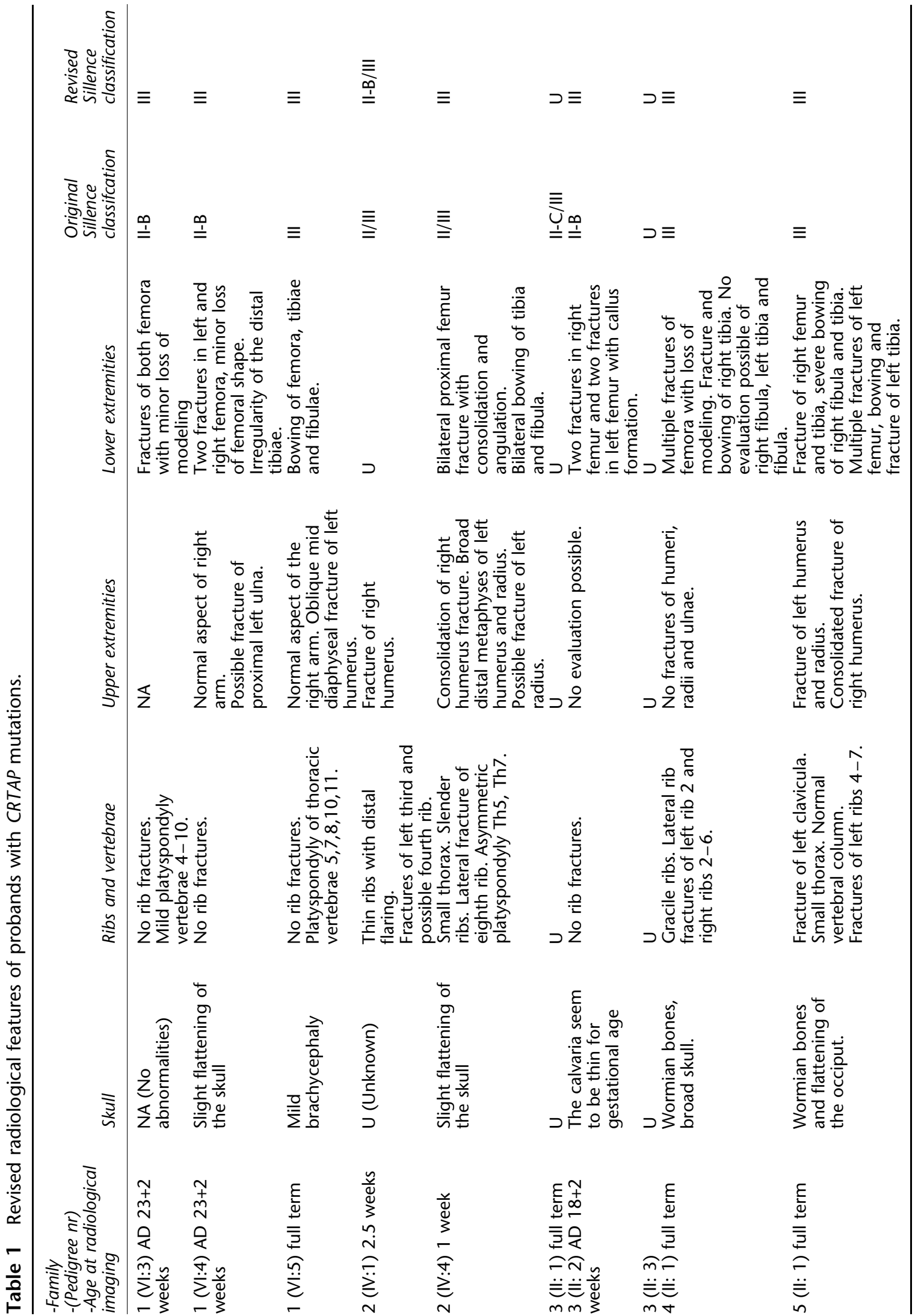


Table 2 CRTAP mutations identified in affected individuals

\begin{tabular}{|c|c|c|c|c|}
\hline Family (pedigree $n r$ ) & Exon/intron & Mutation & Effect of mutation on $m R N A$ & Effect of mutation on protein \\
\hline 1 (Vl:3,Vl:4, Vl:5) & Exon 1 & $\begin{array}{l}\text { c. } 21 \_22 \text { dupGG } \\
\text { c. } 21 \_22 \text { dupGG }\end{array}$ & Not tested & $\begin{array}{l}\text { p.Ala8GlyfsX6 } \\
\text { p.Ala8GlyfsX6 }\end{array}$ \\
\hline $2(\mathrm{IV}: 4)$ & Exon 1 & $\begin{array}{l}\text { c. } 404 \mathrm{delC} \\
\text { c. } 404 \mathrm{delC}\end{array}$ & Not tested & $\begin{array}{l}\text { p.Ser135Thrfs X39 } \\
\text { p.Ser135ThrfsX39 }\end{array}$ \\
\hline 3 (II:1, II:2, II:5) & $\begin{array}{l}\text { Exon1 } \\
\text { Intron1 }\end{array}$ & $\begin{array}{l}\text { c. } 198 C>A \\
\text { c. } 471+2 C>A\end{array}$ & $\begin{array}{l}\text { No effect } \\
\text { Splice error resulting in nonsense-mediated } \\
\text { decay }\end{array}$ & $\begin{array}{l}\text { p.Tyr66X } \\
0 \text {-allele }\end{array}$ \\
\hline $4(I I: 1)$ & Intron 4 & c. $923-2 A>G$ & $\begin{array}{l}\text { Loss of acceptor site and use of } \\
\text { kryptic site resulting in c.923-936del }\end{array}$ & p.Leu308X \\
\hline & $\begin{array}{l}\text { Exon } 1 \\
\text { Exon } 1\end{array}$ & $\begin{array}{l}\text { c. } 38 C>A^{a} \\
\text { c. } 469 A>G^{a}\end{array}$ & $\begin{array}{l}\text { No effect } \\
\text { No effect }\end{array}$ & $\begin{array}{l}\text { p.Ala13Glu } \\
\text { p.Lys157Glu }\end{array}$ \\
\hline $5(\mathrm{II}: 1)$ & Intron 1 & $\begin{array}{l}\text { c. } 471+2 C>A \\
\text { c. } 471+2 C>A\end{array}$ & $\begin{array}{l}\text { Splice error resulting in nonsense-mediated } \\
\text { decay }\end{array}$ & 0-allele \\
\hline
\end{tabular}

${ }^{\mathrm{a}}$ The $\mathrm{c.38} \mathrm{C}>\mathrm{A}$ mutation and the c.469 A>G both reside on the maternal allele.

the femoral epiphysis in proband IV:4 from family 2 did not show a primary cartilaginous dysplasia (see Figure 4). Furthermore, popcorn epiphysis has been reported in a patient with OI due to a COL1A2 mutation, and recently in other patients with OI type III and IV due to COL1A1 and COL1A2 mutations. ${ }^{24,25}$ Popcorn epiphyses were absent in the 4-year-old proband II: 1 from family 5 (see Figure 5 Supplementary Appendix). Considering the above, we hypothesize that popcorn calcifications reflect a disturbance of enchondral ossification, which can occur in CRTAP-related OI as well as in COL1A1/2-related OI.

Importantly, on revision of the available radiographs by a pediatric radiologist, the classification of OI differed in three of the eight cases (Table 2), which stresses the need for evaluation of radiographs by a pediatric radiologist in all cases of OI. It is interesting that a radiological diagnosis of OI type II-B or III was evident in all the affected individuals with CRTAP mutations in the literature, ${ }^{15,22}$ as well as in all our patients. One important difference between OI type II-A, $-\mathrm{B}$ and $-\mathrm{C}$ is the extent and manner of rib involvement. ${ }^{3}$ In OI II-A, the ribs are very broad with contiguous fractures (contiguous beading), whereas in OI II-B, the ribs are rather small, with an irregular course and few fractures, and in OI II-C, which is very rare, varying thickness and discontinuous beading of the ribs are noted. In OI III, rib fractures are less commonly observed. Interestingly, a recurrence rate from $0 \%$ in OI II-A patients ${ }^{4}$ to $7.7 \%$ in OI II-B patients ${ }^{5}$ has been described. It seems that CRTAP mutations cause OI type II-B/III, and it might be that recurrence in OI type II-A is because of gonadal mosaicism of COL1A1/2 mutations only. However, OI type II-B/III can also be caused by COL1A1/2 mutations, which makes differentiation of CRTAP-related OI on the basis of radiological features difficult.

On biochemical testing by electrophoresis, full overmodification of collagen type I was observed comparable with predominantly inherited lethal and severe OI due to collagen type I mutations. However, in some instances, it is possible to discriminate between autosomal recessive and predominantly inherited lethal and severe OI, as in the latter, some normal molecules can be made and chains with normal mobility can be visualized.

In the affected individuals, genomic and complementary DNA sequencing of the COL1A1 and COL1A2 genes was negative. Mutation analysis of CRTAP and LEPRE1 genes identified seven new CRTAP mutations in five families and one new homozygous LEPRE1 mutation in one family. An overview of (new) CRTAP mutations is given in Table 2 and Figure 6 of the Supplementary Appendix. Pathogenicity of the identified mutations was supported by in silico analysis. The c.21_22dupGG and c.404delC mutations in exon 1 are frameshift mutations, leading to a stop codon 6 and 39 amino acids downstream, respectively. The c. $471+2 \mathrm{C}>\mathrm{A}$ mutation is a splice donor-site mutation that changes the second nucleotide in intron 1 , which is the unusual nucleotide, C. (see Supplementary Appendix Figure 1). cDNA sequencing proves that this mutation causes a null allele (see Supplementary Appendix Figure 2). The 198C $>$ A mutation replaces tyrosine at position 66 with a premature termination codon. It is interesting to note that II:1 from family 4 has three mutations. The c.923-2A $>$ G mutation in intron 4 is a splice acceptor site mutation leading to disruption of the normal splicing of exon 5 . The c. $469 \mathrm{~A}>\mathrm{G}$ mutation in exon 1 and the c.38 C $>$ A mutation reside on the same allele. The c.469A $>\mathrm{G}$ mutation is a non-conservative change resulting in a p.Lys157Glu amino acid change and could therefore be considered pathogenic. However, the c.38C $>\mathrm{A}$ missense mutation resulting in p.Ala13Glu might also be pathogenic, as it disturbs the charge distribution in the signal peptide and could lead to retainment of CRTAP in the rER. Currently, functional studies are being conducted to investigate the pathogenicity of these mutations. 
In conclusion, although a diagnosis of OI type II-A seems to point to autosomal dominant OI, in OI types II-B and III, clinical, radiological and biochemical differentiation between autosomal dominant OI caused by collagen type 1 mutations and autosomal recessive OI caused by CRTAP mutations proves difficult. Therefore, it is important to carry out biochemical analysis in combination with preferably genomic and complementary DNA sequencing of the collagen type I genes in individuals affected with lethal or severe OI. Posttranslational overmodification without collagen type I mutations should prompt CRTAP or LEPRE1 mutation analysis. This approach is important for providing accurate recurrence risk, prenatal diagnosis and preimplantation genetic diagnosis. Moreover, therapeutic options might differ in the future. Furthermore, knowledge of the proportion of cases of lethal and severe OI with recurrence due to CRTAP and LEPRE1 mutations will lead to a more accurate estimation of recurrence risk due to germline mutations in the collagen type I genes. It may very well be that the greater part of the empirical recurrence risk in OI type II and III, currently estimated at $6 \%$ for OI type II, ${ }^{9}$ can be attributed to autosomal recessive causes.

\section{Acknowledgements}

We thank the parents for their kind cooperation and we thank clinical geneticist, IJ van der Smagt, at the Department of Clinical Genetics, University Medical Center Utrecht, The Netherlands for his advice.

\section{References}

1 Baljet B: Aspects of the history of osteogenesis imperfecta (Vrolik's syndrome). Ann Anat 2002; 184: 1-7.

2 Sillence DO, Senn A, Danks DM: Genetic heterogeneity in osteogenesis imperfecta. J Med Genet 1979; 16: 101-116.

3 Sillence DO, Barlow KK, Garber AP, Hall JG, Rimoin DL: Osteogenesis imperfecta type II delineation of the phenotype with reference to genetic heterogeneity. Am J Med Genet 1984; 17: $407-423$.

4 Young ID, Thompson EM, Hall CM, Pembrey ME: Osteogenesis imperfecta type IIA: evidence for dominant inheritance. $J$ Med Genet 1987; 24: 386-389.

5 Thompson EM, Young ID, Hall CM, Pembrey ME: Recurrence risks and prognosis in severe sporadic osteogenesis imperfecta. J Med Genet 1987; 24: 390-405.

6 Chu ML, Williams CJ, Pepe G, Hirsch JL, Prockop DJ, Ramirez F: Internal deletion in a collagen gene in a perinatal lethal form of osteogenesis imperfecta. Nature 1983; 304: 78-80.

7 Cohn DH, Starman BJ, Blumberg B, Byers PH: Recurrence of lethal osteogenesis imperfecta due to parental mosaicism for a dominant mutation in a human type I collagen gene (COL1A1). Am J Hum Genet 1990; 46: 591-601.

8 Cohen-Solal L, Bonaventure J, Maroteaux P: Dominant mutations in familial lethal and severe osteogenesis imperfecta. Hum Genet 1991; 87: 297-301.
9 Byers PH, Tsipouras P, Bonadio JF, Starman BJ, Schwartz RC: Perinatal lethal osteogenesis imperfecta (OI type II): a biochemically heterogeneous disorder usually due to new mutations in the genes for type I collagen. Am J Hum Genet 1988; 42: 237-248.

10 Byers PH, Wallis GA, Willing MC: Osteogenesis imperfecta: translation of mutation to phenotype. J Med Genet 1991; 28: $433-442$.

11 Wallis GA, Sykes B, Byers PH, Mathew CG, Viljoen D, Beighton P: Osteogenesis imperfecta type III: mutations in the type I collagen structural genes, COL1A1 and COL1A2, are not necessarily responsible. J Med Genet 1993; 30: 492-496.

12 Rauch F, Glorieux FH: Osteogenesis imperfecta. Lancet 2004; 363: $1377-1385$.

13 Ward LM, Rauch F, Travers R et al: Osteogenesis imperfecta type VII: an autosomal recessive form of brittle bone disease. Bone 2002; 31: $12-18$.

14 Morello R, Bertin TK, Chen Y, Hicks J, Tonachini L, Monticone M. et al. CRTAP is required for Prolyl 3-Hydroxylation and mutations cause recessive osteogenesis imperfecta. Cell 2006; 127: $291-304$

15 Barnes AM, Chang W, Morello R et al: Deficiency of cartilageassociated protein in recessive lethal osteogenesis imperfecta. $N$ Engl J Med 2006; 355: 2757-2764.

16 Cabral WA, Chang W, Barnes AM et al: Prolyl 3-hydroxylase 1 deficiency causes a recessive metabolic bone disorder resembling lethal/severe osteogenesis imperfecta. Nat Genet 2007; 39: 359-365.

17 Marini JC, Cabral WA, Barnes AM, Chang W: Components of the collagen prolyl 3-hydroxylation complex are crucial for normal bone development. Cell Cycle 2007; 6: 1675-1681.

18 Prockop DJ, Constantinou CD, Dombrowski KE et al: Type I procollagen: the gene-protein system that harbors most of the mutations causing osteogenesis imperfecta and probably more common heritable disorders of connective tissue. Am J Med Genet 1989; 34: 60-67.

19 Canty EG, Kadler KE: Procollagen trafficking, processing and fibrillogenesis. J Cell Sci 2005; 118: 1341-1353.

20 Trackman PC: Diverse biological functions of extracellular collagen processing enzymes. J Cell Biochem 2005; 96: 927-937.

21 Korkko J, la-Kokko L, De PA, Nuytinck L, Earley J, Prockop DJ: Analysis of the COL1A1 and COL1A2 genes by PCR amplification and scanning by conformation-sensitive gel electrophoresis identifies only COL1A1 mutations in 15 patients with osteogenesis imperfecta type I: identification of common sequences of null-allele mutations. Am J Hum Genet 1998; 62: 98-110.

22 Baldridge D, Schwarze U, Morello R et al: CRTAP and LEPRE1 mutations in recessive osteogenesis imperfecta. Hum Mutat 2008; 29: $1435-1442$.

23 Spotila LD, Colige A, Sereda L et al: Mutation analysis of coding sequences for type I procollagen in individuals with low bone density. J Bone Miner Res 1994; 9: 923-932.

24 Cole WG, Chan D, Chow CW, Rogers JG, Bateman JF: Disrupted growth plates and progressive deformities in osteogenesis imperfecta as a result of the substitution of glycine 585 by valine in the alpha 2 (I) chain of type I collagen. J Med Genet 1996; 33: 968-971.

25 Obafemi AA, Bulas DI, Troendle J, Marini JC: Popcorn calcifications in osteogenesis imperfecta: incidence, progression and molecular correlation. Am J Med Gen A Sept 2008; 146A: $2725-2732$.

26 Nikkels PGJ: The skeletal system; in Keeling JW, Kong TY (eds): Fetal and Neonatal Pathology, 4 edn. Springer, 2008. pp 770-795.

Supplementary Information accompanies the paper on European Journal of Human Genetics website (http://www.nature.com/ejhg) 